\title{
The two parameter hyperbola problem
}

by

\section{G. KuBA (Wien)}

1. Introduction. Consider a hyperbola

$$
\frac{x^{2}}{a^{2}}-\frac{y^{2}}{b^{2}}=1
$$

in the Euclidian $x, y$-plane with $a, b>0$ and let $R(a, b)$ be the number of lattice points (of the standard lattice $\mathbb{Z}^{2}$ ) "between" the hyperbola and its asymptotes, i.e.,

$$
R(a, b)=\#\left\{(x, y) \in \mathbb{Z}^{2} \mid 0<\frac{x^{2}}{a^{2}}-\frac{y^{2}}{b^{2}} \leq 1\right\} .
$$

The aim of this paper is to develop an asymptotic expansion of $R(a, b)$ in terms of $a$ and $b$ (the major and minor axis of the hyperbola). In [7] we considered the same problem for an ellipse, which turned out to be a pure geometric lattice point problem. In contrast, for hyperbolas the lattice point problem is to a large extent of arithmetic nature since the number $R(a, b)$ is finite if and only if the slope $a / b$ of the asymptote is a rational number. As a consequence, we must assume that $a / b \in \mathbb{Q}$ for the lattice point problem to be well defined. Moreover, the magnitude of $R(a, b)$ will not only depend on the size of $a$ and $b$, but also on the size of the numerator $p$ and the denominator $q$ of the reduced fraction which is equal to $a / b$. Another important difference to the ellipse problem is the fact that the implication $a_{1} \geq a \wedge b_{1} \geq b \Rightarrow R\left(a_{1}, b_{1}\right) \geq R(a, b)$ does not hold for the hyperbola.

In order to avoid expressions including all four quantities $a, b, p, q$ which cannot be interpreted in a meaningful way we will assume $a, b$ to be integers. Thus, we will investigate the behavior of the function $R(a, b)$ for $(a, b) \in \mathbb{N}^{2}$. The choice of $\mathbb{N}^{2}$ as parameter domain can in addition be justified by the fact that $\left\{(a, b) \in \mathbb{R}^{2} \mid a, b>0 \wedge a / b \in \mathbb{Q}\right\}$ is both a null set and a set of first category.

Thus, the objective of the present paper is a proof of the following result. 
THEOREM. For arbitrary positive integers $a, b$ let $d=d(a, b)$ be the greatest common divisor of $a$ and $b, a \sqcap b=\min \{a, b\}, a \sqcup b=\max \{a, b\}$, and

$$
R(a, b)=\#\left\{(x, y) \in \mathbb{Z}^{2} \mid 0<\frac{x^{2}}{a^{2}}-\frac{y^{2}}{b^{2}} \leq 1\right\} .
$$

Then as $a b \rightarrow \infty$,

$$
R(a, b) \sim 2 a b \log \frac{a b}{d}
$$

More precisely, the following asymptotic expansion holds:

$$
R(a, b)=2 a b \log \frac{a b}{d}+(2 \gamma-1) a b+\Delta(a, b),
$$

where $\gamma=0.577215 \ldots$ is Euler's constant and

$$
\begin{aligned}
& \Delta(a, b) \ll \frac{(a b)^{23 / 73}(a \sqcap b)^{50 / 73}}{d^{50 / 73}}(\log d(a \sqcup b))^{461 / 146} \\
& \quad \text { for } d \geq(a \sqcup b)^{19 / 25},
\end{aligned}
$$

(ii) $\quad \Delta(a, b) \ll \frac{(a b)^{23 / 73}(a \sqcup b)^{50 / 73}}{d^{50 / 73}}(\log d(a \sqcap b))^{461 / 146}$

(iii) $\quad \Delta(a, b) \ll \frac{a b}{d^{3 / 2}} \quad$ for $d<(a \sqcap b)^{19 / 25}$.

$$
\text { for }(a \sqcap b)^{19 / 25} \leq d<(a \sqcup b)^{19 / 25},
$$

The $\ll-$ constants are absolute.

2. Applications. In order to illustrate our Theorem, we give some examples of applications. First of all we consider the one parameter case where the hyperbola problem is simply a generalized form of the divisor problem:

Corollary 1. For fixed $p, q \in \mathbb{N}$ and arbitrary $k \in \mathbb{N}$, let $(p ; q)$ be the greatest common divisor of $p$ and $q$ and

$$
R(k)=\#\left\{(x, y) \in \mathbb{Z}^{2} \mid 0<(p x+q y)(p x-q y) \leq p^{2} q^{2} k^{2}\right\} .
$$

Then

$R(k)=2 p q k^{2} \log \left(\frac{p q}{(p ; q)} k\right)+(2 \gamma-1) p q k^{2}+O\left(k^{46 / 73}(\log k)^{461 / 146}\right) \quad(k \rightarrow \infty)$.

Proof. With $(a, b)=(k q, k p)$ we have $d=k(p ; q)$ and $R(k)=R(a, b)$.

Another example where $d \asymp a$ or $d \asymp b$ is given in the following corollary.

Corollary 2. Let $n, m, k$ be positive integers. Then as $k^{n+m} \rightarrow \infty$,

$$
R\left(k^{n}, k^{m}\right)=2(\max \{n, m\}) k^{n+m} \log k+(2 \gamma-1) k^{n+m}+\Delta_{k, n, m},
$$

where 


$$
\begin{aligned}
& \Delta_{k, n, m} \ll k^{\frac{23}{73}(n+m)}((n+m)(\log k))^{461 / 146} \\
& \text { if } 25(\min \{n, m\}) \geq 19(\max \{n, m\}),
\end{aligned}
$$

and

$$
\begin{aligned}
& \Delta_{k, n, m} \ll k^{\frac{23}{73}(n+m)+\frac{50}{73}|n-m|}((n+m)(\log k))^{461 / 146} \\
& \text { if } 25(\min \{n, m\})<19(\max \{n, m\}) .
\end{aligned}
$$

The examples in Corollaries 1 and 2 belong to the most likely case where the greatest common divisor $d$ of $a$ and $b$ is large for large $a$ and $b$. In the following corollary we consider an extreme instance of the case where $d$ is bounded.

COROLlary 3. For relatively prime $a, b \in \mathbb{N}$,

$$
R(a, b)=2 a b \log a b+O(a b) .
$$

In this case we cannot determine an estimate of the error term $\Delta(a, b)$ which is better than the trivial $O(a b)$.

3. Proof of the Theorem. Evaluation of the main term. In order to calculate $R(a, b)$ it is sufficient to count all lattice points in the domain

$$
D(a, b):=\left\{(x, y) \in \mathbb{R}^{2} \mid x, y>0 \wedge 0<\frac{x^{2}}{a^{2}}-\frac{y^{2}}{b^{2}} \leq 1\right\} .
$$

Then we have

$$
R(a, b)=4 \#\left(D(a, b) \cap \mathbb{Z}^{2}\right)+2 a .
$$

The main idea now is to count the lattice points in $D(a, b)$ along lines parallel to the asymptote $x / a-y / b=0$. For abbreviation, we put

$$
\hat{a}=\frac{a}{d} \quad \text { and } \quad \hat{b}=\frac{b}{d},
$$

where $d$ is the greatest common divisor of $a$ and $b$. Then the "counting" lines are all lines $g_{n}$,

$$
g_{n}: \hat{b} x-\hat{a} y=n \quad \text { with } n=1,2, \ldots, a b / d .
$$

(For $n=a b / d$ the vertex $(a, 0)$ of the hyperbola is the only lattice point on $g_{n}$ in $\overline{D(a, b)}$.)

Let $S_{n}$ be the intersection point of $g_{n}$ with the $x$-axis and $T_{n}$ be the intersection point of $g_{n}$ with the hyperbola $(b x-a y)(b x+a y)=a^{2} b^{2}$. Then $S_{n}=(n / \hat{b}, 0)$ and the $x$-coordinate $x_{n}$ of $T_{n}$ is given by

$$
x_{n}=\frac{1}{2 b}\left(d n+\frac{a^{2} b^{2}}{d n}\right) .
$$


Now we can write

$$
R_{1}(a, b):=\#\left(D(a, b) \cap \mathbb{Z}^{2}\right)=\sum_{1 \leq n \leq a b / d} r_{n},
$$

where $r_{n}$ is the number of all lattice points on the line $g_{n}$ between $S_{n}$ (excluded) and $T_{n}$ (included).

In order to calculate $r_{n}$, let $x=x(n)$ be the unique solution of the congruence

$$
\hat{b} x \equiv n(\bmod \hat{a})
$$

in the interval $1 \leq x \leq \hat{a}$. Then for every $x \in x(n)+\hat{a} \mathbb{Z}$ there is exactly one integer $y$ with $(x, y) \in g_{n}$. Of course, $x(n)$ only depends on the residue class $n+\hat{a} \mathbb{Z}$, so we will write $x(n+\hat{a} \mathbb{Z})$ instead of $x(n)$. Then we have

$$
\begin{aligned}
r_{n} & =\#\left\{k \in \mathbb{Z} \mid n / \hat{b}<x(n+\hat{a} \mathbb{Z})+k \hat{a} \leq x_{n}\right\} \\
& =\left[\frac{x_{n}-x(n+\hat{a} \mathbb{Z})}{\hat{a}}\right]-\left[\frac{n}{\hat{a} \hat{b}}-\frac{x(n+\hat{a} \mathbb{Z})}{\hat{a}}\right] .
\end{aligned}
$$

([] are the Gauss brackets.)

Now let $\psi(\cdot)$ be defined by

$$
\psi(z)=z-[z]-1 / 2 \quad(z \in \mathbb{R}) .
$$

Then we compute

$$
R_{1}(a, b)=\sum_{1 \leq n \leq a b / d}\left(\frac{1}{2 \hat{a} b}\left(d n+\frac{a^{2} b^{2}}{d n}\right)-\frac{n}{\hat{a} \hat{b}}\right)+\Psi_{1}(a, b)-\Psi_{2}(a, b),
$$

where

$$
\begin{aligned}
& \Psi_{1}(a, b)=\sum_{1 \leq n \leq a b / d} \psi\left(\frac{n}{\hat{a} \hat{b}}-\frac{x(n+\hat{a} \mathbb{Z})}{\hat{a}}\right), \\
& \Psi_{2}(a, b)=\sum_{1 \leq n \leq a b / d} \psi\left(\frac{x_{n}-x(n+\hat{a} \mathbb{Z})}{\hat{a}}\right) .
\end{aligned}
$$

The calculation of the main term of $R_{1}(a, b)$ is straightforward. We make use of the well-known formulas (for the second see Fricker [2])

$$
\sum_{1 \leq n \leq N} n=N(N+1) / 2
$$

and

and obtain

$$
\sum_{1 \leq n \leq N} \frac{1}{n}=\log N+\gamma+\frac{1}{2 N}+O\left(N^{-2}\right)
$$

$$
R_{1}(a, b)=\frac{a b}{2} \log \frac{a b}{d}+\gamma \frac{a b}{2}-\frac{a b}{4}+\Psi_{1}(a, b)-\Psi_{2}(a, b)+O(1) .
$$


In order to calculate $\Psi_{1}(a, b)$, we introduce two new parameters $A$ and $B$. Let $A, B$ be integers satisfying $A \hat{a}+B \hat{b}=1$. Then for every integer $n$,

$$
x(n+\hat{a} \mathbb{Z}) \equiv B n(\bmod \hat{a}) .
$$

Now, let $y=y(n+\hat{b} \mathbb{Z})$ be the unique solution of the congruence $\hat{a} y \equiv n$ $(\bmod \hat{b})$ in the interval $1 \leq y \leq \hat{b}$. Then for every $n$,

$$
y(n+\hat{b} \mathbb{Z}) \equiv A n(\bmod \hat{b}),
$$

and we obtain

$$
\frac{n}{\hat{a} \hat{b}}-\frac{x(n+\hat{a} \mathbb{Z})}{\hat{a}} \equiv \frac{y(n+\hat{b} \mathbb{Z})}{\hat{b}}(\bmod 1) .
$$

Since $\psi(\cdot)$ is a periodic function with period 1 , the sum $\Psi_{1}(a, b)$ becomes a sum over a complete set of residues $\bmod \hat{b}$, repeated $a$ times. We obtain

$$
\Psi_{1}(a, b)=\sum_{1 \leq n \leq a \hat{b}} \psi\left(\frac{y(n+\hat{b} \mathbb{Z})}{\hat{b}}\right)=a \sum_{1 \leq n \leq \hat{b}}\left(\psi\left(\frac{n}{\hat{b}}\right)\right)=-\frac{a}{2} .
$$

Thus we derive

$$
R(a, b)=2 a b \log \frac{a b}{d}+(2 \gamma-1) a b-4 \Psi_{2}(a, b)+O(1) .
$$

The estimation of $\Psi_{2}(a, b)$ now concludes the proof of the Theorem.

4. Estimation of $\Psi_{2}$. First, we want to get rid of the deranging term $x(n+\hat{a} \mathbb{Z}) / \hat{a}$ in $\Psi_{2}(a, b)$. We substitute $x(n+\hat{a} \mathbb{Z}) / \hat{a}$ by $B n / \hat{a}$. Then we have

$$
\Psi_{2}(a, b)=\sum_{1 \leq n \leq a b / d} \psi\left(\frac{1}{2 \hat{a} b}\left(d n+\frac{a^{2} b^{2}}{d n}\right)-\frac{B n}{\hat{a}}\right) .
$$

In order to compute this sum, we divide $n$ into residue classes mod $\hat{a}$ as well as into residue classes mod $\hat{b}$ and make use of the equation

$$
\sum_{1 \leq n \leq a b / d} F(n)=\sum_{1 \leq m \leq \hat{a}} \sum_{0 \leq l<b} F(l \hat{a}+m)=\sum_{1 \leq m \leq \hat{b}} \sum_{0 \leq l<a} F(l \hat{b}+m),
$$

which holds for every function $F$ defined on $\{1,2, \ldots, a b / d\}$.

Furthermore, we note that

$$
\frac{1}{2 \hat{a} b}\left(d n+\frac{a^{2} b^{2}}{d n}\right)-\frac{B n}{\hat{a}}=\frac{1}{2 a \hat{b}}\left(\frac{a^{2} b^{2}}{d n}-d n\right)+\frac{A n}{\hat{b}} .
$$

Therefore

$$
\Psi_{2}(a, b)=\sum_{1 \leq m \leq \hat{a}} \Psi_{m}^{*}(a, b)=\sum_{1 \leq m \leq \hat{b}} \Psi_{m}^{* *}(a, b)
$$


where

$$
\Psi_{m}^{*}(a, b)=\sum_{0 \leq l<b} \psi\left(f_{m}(l)\right) \text { and } \Psi_{m}^{* *}(a, b)=\sum_{0 \leq l<a} \psi\left(g_{m}(l)\right),
$$

with

$$
\begin{aligned}
& f_{m}(t)=\left(\frac{1}{2 \hat{a} b}\left(\frac{a^{2} b^{2}}{d \hat{a} t+d m}+d \hat{a} t+d m\right)-\frac{B m}{\hat{a}}\right), \\
& g_{m}(t)=\left(\frac{1}{2 a \hat{b}}\left(\frac{a^{2} b^{2}}{d \hat{b} t+d m}-d \hat{b} t-d m\right)+\frac{A m}{\hat{b}}\right) .
\end{aligned}
$$

In order to establish clauses (i) and (ii), we make use of an essential tool from Huxley's "Discrete Hardy-Littlewood Method" in the shape presented in Huxley [3] and [4]. The following lemma is a combination of Huxley [4], Theorem 3 and Theorem 4:

Lemma 1. Let $M, M^{\prime}$ and $T$ be positive real parameters satisfying $M \leq$ $M^{\prime}<2 M$ and $M \leq C_{1} T^{83 / 146}(\log T)^{-63 / 292}$ with a constant $C_{1}$. Furthermore, let $F(t)$ be a four times continuously differentiable function on $1 \leq t \leq 2$ satisfying

$F^{\prime}(t), F^{\prime \prime}(t), F^{(3)}(t), F^{\prime}(t) F^{(3)}(t)-3\left(F^{\prime \prime}(t)\right)^{2}, F^{\prime \prime}(t) F^{(4)}(t)-3\left(F^{(3)}(t)\right)^{2} \neq 0$

for all $1 \leq t \leq 2$. Then

$$
\sum_{M \leq k \leq M^{\prime}} \psi\left(\frac{T}{M} F\left(\frac{k}{M}\right)\right) \ll T^{23 / 73}(\log T)^{315 / 146} .
$$

The $\ll$-constant depends on $C_{1}$ and on the range of values taken by the derivatives of $F$.

To verify (iii), we use van der Corput's classical estimate of $\psi$-sums:

Lemma 2 (see van der Corput [1]). Let $f$ be a real-valued function, twice continuously differentiable on $[a, b] \subset \mathbb{R}$. Furthermore, let $f^{\prime \prime}$ be monotonic and nonzero on $[a, b]$. Then

$$
\sum_{a \leq k \leq b} \psi(f(k)) \ll \int_{a}^{b}\left|f^{\prime \prime}(t)\right|^{1 / 3} d t+\left|f^{\prime \prime}(a)\right|^{-1 / 2}+\left|f^{\prime \prime}(b)\right|^{-1 / 2},
$$

where the $\ll$-constant is absolute.

In order to estimate $\Psi_{m}^{*}(a, b)$ for every $m=1, \ldots, \hat{a}$, we put $\beta=b-2$ and

$$
f(t):=f_{m}(t)=\frac{d}{2 b} t+\frac{1}{2} \cdot \frac{a b}{\hat{a} t+m}+\frac{d^{2} m}{2 a b}-\frac{B m}{\hat{a}} \quad(0<t \leq \beta) .
$$


Then

$$
\Psi_{m}^{*}(a, b)=\sum_{l=1}^{\beta} \psi(f(l))+O(1)
$$

We note that

$$
\begin{gathered}
f^{\prime}(t)=\frac{d}{2 b}-\frac{\hat{a} a b}{2(\hat{a} t+m)^{2}}, \quad f^{\prime \prime}(t)=\frac{\hat{a}^{2} a b}{(\hat{a} t+m)^{3}}, \\
f^{(3)}(t)=-\frac{3 \hat{a}^{3} a b}{(\hat{a} t+m)^{4}}, \quad f^{(4)}(t)=\frac{12 \hat{a}^{4} a b}{(\hat{a} t+m)^{5}},
\end{gathered}
$$

and observe that $f^{\prime}(t), f^{\prime \prime}(t), f^{(3)}(t) \neq 0$ for all $\left.\left.t \in\right] 0, \beta\right]$. Furthermore, we see that for $0<t \leq \beta$,

$$
f^{\prime}(t) f^{(3)}(t)-3\left(f^{\prime \prime}(t)\right)^{2}=-\frac{3 a \hat{a}^{3}\left(a \hat{a} b^{2}+d(\hat{a} t+m)^{2}\right)}{2(\hat{a} t+m)^{6}} \neq 0,
$$

and

$$
f^{\prime \prime}(t) f^{(4)}(t)-3\left(f^{(3)}(t)\right)^{2}=-\frac{15 a^{2} \hat{a}^{6} b^{2}}{(\hat{a} t+m)^{8}} \neq 0 .
$$

The sum $\Psi_{m}^{*}(a, b)$ may now be written as

$$
\Psi_{m}^{*}(a, b)=\sum_{1 \leq j<J} S_{j}+O(1)
$$

with

$$
S_{j}=\sum_{M_{j} \leq k<M_{j+1}} \psi(f(k)) \quad(1 \leq j<J)
$$

where $\left(M_{j}\right)_{1 \leq j \leq J}$ is a geometric series, $M_{j}=2^{j} M_{1}, M_{J}=\beta+1$, and $1 / 2 \leq M_{1}<1$.

Now we apply Lemma 1 to each $S_{j}$. Let

$$
M=M_{j}, \quad M^{\prime}=-[-2 M]-1, \quad T=d b \quad \text { and } \quad F(u)=\frac{M}{T} f(M u) .
$$

Then $F(u)$ satisfies all conditions of Lemma 1. In addition we have $\left|F^{(r)}(u)\right|$ $\ll 1$ for $1 \leq u \leq 2$ and $r=1,2,3,4$.

Furthermore, we note that for $b \leq d^{83 / 63-\varepsilon}$ (which is true if $d \geq b^{19 / 25}$ ) the condition $M \leq C_{1} T^{83 / 146}(\log T)^{-63 / 292}$ is fulfilled for all $M \in$ $\left\{M_{1}, \ldots, M_{J}\right\}$.

Thus, all conditions of Lemma 1 are satisfied, and we obtain

$$
S_{j} \ll(d b)^{23 / 73}(\log d b)^{315 / 146} \quad(j=1, \ldots, J-1) .
$$

Observing that $J \ll \log b$ we derive

$$
\Psi_{m}^{*}(a, b) \ll(d b)^{23 / 73}(\log d b)^{1+315 / 146},
$$


and this leads to

$$
\Psi_{2}(a, b) \ll \frac{a}{d}(d b)^{23 / 73}(\log d b)^{461 / 146}=\frac{a b^{23 / 73}}{d^{50 / 73}}(\log d b)^{461 / 146},
$$

provided that $d \geq b^{19 / 25}$.

The sums $\Psi_{m}^{* *}(a, b)$ from the second representation of $\Psi_{2}(a, b)$ can be treated in an analogous way. We derive

$$
\Psi_{m}^{* *}(a, b) \ll(d a)^{23 / 73}(\log d a)^{461 / 146} \quad \text { for } d \geq a^{19 / 25} .
$$

Consequently, we also have

$$
\Psi_{2}(a, b) \ll \frac{a^{23 / 73} b}{d^{50 / 73}}(\log d a)^{461 / 146} \quad \text { if } d \geq a^{19 / 25} .
$$

Both estimates put together yield clauses (i) and (ii) of the Theorem.

The estimation of $\Psi_{2}(a, b)$, with the help of van der Corput's method (Lemma 2), is straightforward. We obtain

$$
\Psi_{2}(a, b)=\sum_{1 \leq m \leq \hat{a}} \Psi_{m}^{*}(a, b) \ll \frac{a b^{1 / 3}}{d^{2 / 3}} \log \frac{a b}{d}+\frac{a b}{d^{3 / 2}},
$$

and

$$
\Psi_{2}(a, b)=\sum_{1 \leq m \leq \hat{b}} \Psi_{m}^{* *}(a, b) \ll \frac{a^{1 / 3} b}{d^{2 / 3}} \log \frac{a b}{d}+\frac{a b}{d^{3 / 2}} .
$$

We combine both estimates and substitute $\log \frac{a b}{d}$ by $(a b)^{\varepsilon}$. Then as $a b \rightarrow \infty$,

$$
\Psi_{2}(a, b) \ll \frac{(a b)^{1 / 3+\varepsilon}(a \sqcap b)^{2 / 3}}{d^{2 / 3}}+\frac{a b}{d^{3 / 2}} .
$$

Note that the second term dominates the first if and only if $d(a \sqcap b)^{6 \varepsilon / 5}<$ $(a \sqcup b)^{4 / 5-6 \varepsilon / 5}$. And this condition is surely fulfilled for $d<(a \sqcap b)^{19 / 25}$ if we put $\varepsilon=\frac{5}{12}\left(\frac{4}{5}-\frac{19}{25}\right)$.

This proves clause (iii) of the Theorem.

Remark. A direct (and better) estimation of the sum $\Psi_{2}(a, b)$ without splitting up the interval of summation $1 \leq n \leq a b / d$ into residue classes (as in Huxley and Watt [6], p. 162) seems impracticable. Huxley had a similar difficulty in the Corrigenda: "Exponential sums and lattice points II" [5]. What makes the problem difficult is the deranging term $B n / \hat{a}$. Even van der Corput's method (Lemma 1 ) where linear terms are negligible is of no use because the linear term $B n / \hat{a}$ contains the parameter $B$ from $A \hat{a}+B \hat{b}=1$. Obviously, the parameter $B$ depends uncontrollably on the basic parameters $a, b, d$. The only way to get rid of this problem is, as we have done it, to sum over subintervals where the term $B n / \hat{a}$ is constant modulo 1 . 


\section{References}

[1] J. G. van der Corput, Zahlentheoretische Abschätzungen mit Anwendungen auf Gitterpunktsprobleme, Math. Z. 17 (1923), 250-259.

[2] F. Fricker, Einführung in die Gitterpunktlehre, Birkhäuser, Basel, 1982.

[3] M. N. Huxley, Exponential sums and lattice points, Proc. London Math. Soc. (3) 60 (1990), 471-502.

[4] —, Exponential sums and lattice points II, ibid. 66 (1993), 279-301.

[5] —, Corrigenda: "Exponential sums and lattice points II", ibid. 68 (1994), 264.

[6] M. N. Huxley and N. Watt, The number of ideals in a quadratic field, Proc. Indian Acad. Sci. (Math. Sci.) 104 (1994), 157-165.

[7] G. Kuba, The two parameter ellipse problem, Math. Slovaca 44 (1994), 585-593.

Institut für Mathematik u.a.St.

Universität für Bodenkultur

Gregor Mendel-Straße 33

A-1180 Wien, Austria

E-mail: kuba@edv1.boku.ac.at

Received on 23.6.1995

and in revised form on 11.12.1995 